\title{
Cypriot Patients with Inflammatory Bowel Disease and Quality Of Life
}

\author{
Maria Tsoukka $^{1,2}$, Eleni Jelastopulu ${ }^{1,3}$, George Charalambous ${ }^{1,4}$ and Panagiota Evangelou ${ }^{5}$ \\ ${ }^{1}$ Postgraduate Program Health Management, Frederick University, Nicosia, Cyprus \\ ${ }^{2}$ General Hospital of Larnaca, Larnaca, Cyprus \\ ${ }^{3}$ Department of Public Health, School of Medicine, University of Patras, Greece \\ ${ }^{4}$ General Hospital of Athens "Hippocratio", Athens, Greece \\ ${ }^{5}$ Graduate program Medical School, University of Cyprus
}

Received: October 07,2017; Published: October 13, 2017

*Corresponding author: Maria Tsoukka, Kalavardon No. 16, Dromolaxia, 7020 Larnaca, Cyprus

\begin{abstract}
The aim of the present study was to investigate the Quality of Life of patients suffering from IBD and the factors that might be affecting it. The idiopathic Inflammatory Bowel Diseases (IBD) are chronic nosological entities negatively affecting the Quality Of Life of patients suffering from IBD in relation to the total population. Evaluating their Quality of Life is the basic constitution for evaluating their needs and for designing the suitable health care system to be provided. In the province of Larnaca the SIBDQ questionnaire translated in the Greek language was used to evaluate 100 patients suffering from Ulcerative Colitis and Crohn's disease. We studied the Quality of Life as a dependent variable correlated with the independent variables that are the demographic and general characteristics of this patient group.
\end{abstract}

\section{Introduction}

The Idiopathic Inflammatory Bowel Diseases (IBD) are nosological entities that are characterized by yearly recurrent immune activations and inflammation of the gastrointestinal tube. They are accompanied by relapses and remissions and are directly affecting the patients' lives [1].

Investigating the factors that might affect the Quality of Life of IBD like any other chronic disease constitutes an extreme useful procedure as health systems of all developed countries have made a turn to higher standards of health care systems that corresponds to the needs and expectations of all health professionals [2]. Recognizing the parameters and characteristics of patients related to an unfavorable or better quality of life, creates the necessary conditions that can allow to design properly the provided health care system [3].

\section{Materials and Methods}

The current study constitutes of a population sample of 100 patients suffering from Inflammation Bowel Diseases being Ulcerative Colitis 60\% and Crohn's disease 40\%. All of them being examined at the Endoscopic Unit of district hospitals in Cyprus. For evaluating their quality of Life a partial differentiation of the Greek translation of Short Inflammatory Bowel Disease Questionnaire (SIBDQ) [4], was used. Questionnaires were handed out to patients in personal by the researcher. For the statistic analysis of the research data was used regtration and the statistical software program IBM SPSS Statistics v.20 was used with a minimum level of statistical significance of $\mathrm{p} \leq 0.05$.

\section{Results}

Table 1 presents Quality of Life as a dependent variable related to the independent variables that are the demographic characteristics and the general characteristics of IBD patients. The statistical analysis shows that gender ( $p$ value $=0.32)$, age $(p$ value $=0.21)$, education ( $p$ value $=0.14)$, family status of single persons ( $p$ value $=0.32)$, divorced $(p$ value $=0.54)$, smoking ( $p$ value $=0.91)$ and in relation with the fact if patients underwent a surgery or not $(p$ value $=0.72$ ), have no statistic significant difference leading to the conclusion that do not play any important role in the Quality of Life of these patients. Statistical important difference was recorded, with the score of Quality of Life being increased in Ulcerative Colitis in relation to Crohn's disease ( $p$ value $=0.032$ ), in less duration of the disease ( $p$ value $=0.0031$ ) and in patients that stated themselves as self-employed ( $p$ value $=0.011$ ) in relation with other professions. 


\section{Discussion}

Regarding the results of the current study, that the score of Quality of Life increases for patients with Ulcerative Colitis, consequently Quality of Life in patients with Crohn's is poorer, might be due to the more severe symptomatology and the more severe complications of Crohn's disease thus consequently the larger usage of the health system of these patients in relation to those who suffer from Ulcerative Colitis. In the results of the current study it's also important to note that the score of Quality of Life increases for those of who have had a shorter duration of the disease. This result could be interpreted by the evolution of the disease, the fact of noncompliance of medication/treatment but also the inefficiency of medication. Other reasons that may be a factor leading to this result is that with the passing of time patient's worries are increased as they are related to uncertainty about the development of their disease, the effects of medication, their energy level, the possibility surgery they might need and as a result perhaps the need of orifice and bag, the fact that they might be a burden for others, loss of bowel control, pain, suffering as well as the possibility of getting cancer. Another research finding in this study it was that the score of Quality of Life in patients with IBD it's different in the sector of work, with statistical difference and increased Quality of Life of those who are self employed in relation to the rest who work in the public and private sector as those to those who are unemployed. These findings are possibly related with a research taken place in Greece in 1181 patients with IBD by Viazis et al. [5] where results in the field productivity influence pointed out that problems related with the symptoms of IBD found to intervene in the ability of work at $40 \%$ of patients of this study with more than half $57 \%$ taking a sick leave due to the problems related with their disease or due to the time they have to spend at health care units. Lastly $32 \%$ of patients never informed their employers or their colleagues regarding their disease either because they believed it's a private matter or either from fear of probable negative consequences. It is also possible that the relation of these diseases advocate with negative prejudices along with the feeling of being stigmatized [6]. Maybe all reasons mentioned above contribute to the fact that self-employed patients with IBD have an increased quality of Life in relation to patients that work in other sectors. The independence that the occupation provides to patients probably leads to this increase in their Quality of Life.

\section{References}

1. Baumgart DC, Carding SR (2007) Inflammatory bowel disease: Cause and immunobiology. Lancet 369:1627-1640.

2. Papanicolaou B (2007) Quality of Life in Health care: Principles, Methods and applications. Editions Papazisi Athens.

3. Yiakoumidakis K, Kongoulis D, Elevsiniotis I, Mprokalaki Bananoudaki H (2011) Qualitiy of Life in Patients with Inflammatory Bowel Disease. Nursing 50(1): 95-103.

4. Irvine EJ, Zhou Q Thompson AK (1996) The short Inflammatory Bowel Disease Questionnaire: a quality of life instrument for community physicians managing Inflammatory Bowel Disease 91(8): 1571-8.

5. Viazis N, Mantzaris G, Karmiris K, Polymeros D, Kouklakis G, et al. (2013) Inflammatory bowel disease: Greek patients' perspective on quality of life, information on the disease, work productivity and family support. Annals of Gastroenterology 26(1): 52-58.

6. Sajadinejad MS, Asgari K, Molavi H, Kalantari M, Padibi (2012) P Psychological Issues in Inflammatory Bowel Disease: An Overview Academic Editor: P. Gionchetti Review Article 2012.

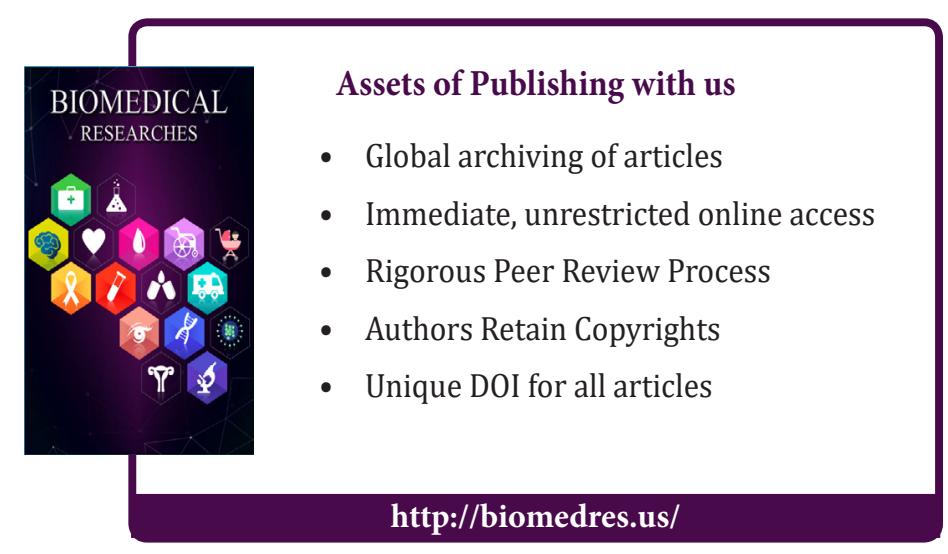

